Konzepte der Kulturwissenschaften 


\section{Konzepte \\ der Kulturwissenschaften}

Theoretische Grundlagen - Ansätze Perspektiven

Herausgegeben von

Ansgar Nünning und Vera Nünning

Verlag J. B. Metzler

Stuttgart - Weimar 
Bibliografische Information Der Deutschen Bibliothek

Die Deutsche Bibliothek verzeichnet diese Publikation in der Deutschen Nationalbibliografie; detaillierte bibliografische Daten sind im Internet über <http://dnb.ddb.de> abrufbar.

ISBN 978-3-476-01737-6

ISBN 978-3-476-02745-0 (eBook)

DOI 10.1007/978-3-476-02745-0

Dieses Werk einschließlich aller seiner Teile ist urheberrechtlich geschützt. Jede Verwertung außerhalb der engen Grenzen des Urheberrechtsgesetzes ist ohne Zustimmung des Verlages unzulässig und strafbar. Das gilt insbesondere für Vervielfältigungen, Übersetzungen, Mikroverfilmungen und die Einspeicherung und Verarbeitung in elektronischen Systemen.

(C) 2003 Springer-Verlag GmbH Deutschland

Ursprünglich erschienen bei J.B. Metzlersche Verlagsbuchhundlung und Carl Ernst Poeschel

Verlag GmbH in Stuttgart 2003

www.metzlerverlag.de

info@metzlerverlag.de 


\section{Inhaltsverzeichnis}

1. Kulturwissenschaften: Eine multiperspektivische Einführung in einen interdisziplinären Diskussionszusammenhang (Vera Nünning

und Ansgar Nünning)

1. Produktive Grenzüberschreitungen, Internationalität,

Perspektivenvielfalt und Pluralisierung der kulturwissen-

schaftlichen Landschaft

2. Schwerpunkte und Ziele dieses Bandes - und was er nicht will ...... 10

2. Kulturbegriffe und Kulturtheorien (Claus-Michael Ort) ........................ 19

1. Vom normativen Kulturbegriff zur Kulturtheorie ............................. 19

1.1 'Kultur< vs. >Natur< ‘......................................................... 19

1.2 'Kultur vs. `Zivilisation ‘....................................................... 21

1.3 ’Kultur vs. `Gesellschaft ‘................................................... 22

2. Sozialtheorie als Kulturtheorie:

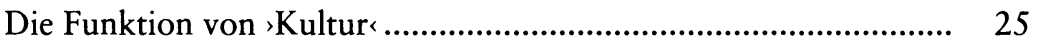

2.1 Die wissenssoziologische Generalisierung der Kultursoziologie ........................................................................ 25

2.2 Systemtheoretische Kultur- und Wissenssoziologie ................. 28

3. Kulturtheorie als Zeichentheorie ................................................... 31

3.1 'Kultur als 'Spracher - -Kultur als Abstraktion .................... 31

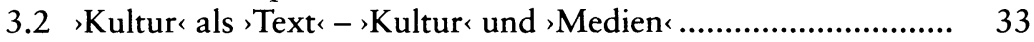

3. Kultursemiotik (Roland Posner) …................................................... 39

1. Begriff und Aufgabe ...................................................................... 39

2. Zeichensysteme in einer Kultur: Prozesse, Codes und Medien ........ $\quad 40$

2.1 Prozesse .......................................................................... 40

2.2 Codes .............................................................................. 42

2.3 Medien ............................................................................... 43

3. Kulturen als Zeichensysteme: Gesellschaft, Zivilisation,

Mentalität

3.1 Soziale Kultur (Gesellschaft): Zeichenbenutzer ....................... 49

3.2 Materiale Kultur (Zivilisation): Texte ..................................... 50

3.3 Mentale Kultur (Mentalität): Codes ........................................ 53

3.4 Der semiotische Zusammenhang von sozialer, materialer und mentaler Kultur 
VI Inhalt

4. Kulturmechanismus und Kulturwandel .................................... 55

4.1 Kultur als System von Texten ............................................ 55

4.2 Kultur als System von Codes .............................................. 58

4.3 Kultur als kollektives Gedächtnis ...................................... 64

5. Zur institutionellen Verankerung der Kultursemiotik ................... 66

4. Literaturwissenschaft als Kulturwissenschaft (Wilhelm Voßkamp) $\ldots . . . \quad 73$

1. Einleitung ..................................................................... 73

2. Wissenschaftsgeschichte des Zusammenhangs von Literatur- und Kulturwissenschaften .

3. Systematische Aspekte des Zusammenhangs

von Literatur- und Kulturwissenschaft

3.1 Literarische Texte als Gegenstände der kulturellen Selbstwahrnehmung

3.2 Medien und Medialität als konstitutive Elemente kultureller Kommunikation

3.3 Rekonstruktion und Konstruktion der Geschichte der Literatur als historisches Problem des Zusammenhangs von Literaturwissenschaft und Kulturwissenschaft

4. Zusammenfassung

5. Kulturanthropologie (Doris Bachmann-Medick)

1. Kurze Geschichte der Kulturanthropologie

2. Interpretative Kulturanthropologie - Kultur als Text ................... 89

3. Performative Kulturanthropologie ......................................... 91

4. Writing Culture .................................................................. 93

5. Kulturanthropologie im Spannungsfeld interkultureller Konfliktdynamik

6. Kulturanthropologie und globale Vernetzungen .......................... 98

7. Kulturanthropologie an den Schnittflächen verschiedener Disziplinen

8. Anthropologie der Kultur - Neigung zum Kulturalismus

6. Historische und literarische Anthropologie (Harald Neumeyer) ......... 108

1. Historische Anthropologie ....................................................... 108

1.1 Ausgangspunkte

1.2 Geschichtswissenschaft, Anthropologie/Ethnologie und Kulturanthropologie in den USA

1.3 Historische Anthropologie in Deutschland .......................... 111

1.4 Institutionelle Verankerung .............................................. 112

1.5 Inhalte, Methoden und Probleme .................................... 114 
2. Literarische Anthropologie ......................................................... 117

2.1 Ausgangspunkte ................................................................ 117

2.2 Der Boom der literarischen Anthropologie in der ersten Hälfte der 1990er Jahre

2.3 Das Ende der literarischen Anthropologie? ............................. 122

2.4 Institutionelle Verankerung ................................................. 125

2.5 Probleme und Perspektiven .................................................. 126

7. New Historicism, Cultural Materialism und Cultural Studies (Moritz Baßler) ........................................................................... 132

1. Standortbestimmung ................................................................ 132

2. Was macht der New Historicist? .................................................. 133

3. Entstehung und Einflüsse ............................................................. 137

3.1 Raymond Williams' Cultural Materialism ............................. 138

3.2 Michel Foucaults Diskursanalyse ............................................ 140

3.3 Clifford Geertz' Konzept der ’dichten Beschreibung '.............. 142

3.4 Paul de Mans `Allegorien des Lesens ‘ ...................................... 143

4. Poetik der Kultur und die Anekdote .............................................. 144

5. Probleme der methodischen Fundierung ...................................... 146

6. Die textuelle Option ................................................................ 148

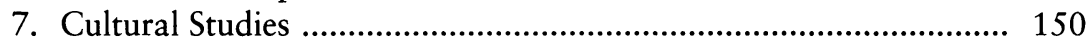

8. Kollektives Gedächtnis und Erinnerungskulturen (Astrid Erll) ............. 156

1. Einleitung: Ein sneues Paradigma und seine Ursachen ................... 156

2. Die `Erfindung des kollektiven Gedächtnisses:

Maurice Halbwachs und Aby Warburg .......................................... 158

2.1 Maurice Halbwachs: Mémoire collective ............................... 158

2.2 Aby Warburg: Das ssoziale Gedächtnis‘ ................................ 161

3. Der Beitrag der Einzeldisziplinen zum Forschungsfeld 'kollektives Gedächtnis und Erinnerungskulturen ‘....................... 164

3.1 Psychologie und Kognitionswissenschaft ............................ 164

3.2 Geschichtswissenschaft ...................................................... 166

3.3 Kunst- und Literaturwissenschaft ......................................... 168

4. Aleida und Jan Assmanns Theorie des kulturellen

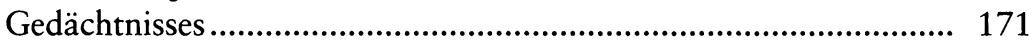

4.1 Kommunikatives und kulturelles Gedächtnis ......................... 171

4.2 Kulturelles Gedächtnis, Schrift und politische Identität .......... 173

4.3 Gedächtnis als ars und vis, Funktionsgedächtnis und Speichergedächtnis

5. Formen von Gedächtnis und Erinnerung auf kollektiver Ebene: Anregungen zur begrifflichen Differenzierung

5.1 Kollektives Gedächtnis, Akte kollektiver Erinnerung und Erinnerungskulturen 
VIII Inhalt

5.2 Symbolische Formen und kulturelle Dimensionen des Gedächtnisses

5.3 Explizite und implizite Systeme des kollektiven

Gedächtnisses

6. Das Forschungsfeld >kollektives Gedächtnis und Erinnerungs-

kulturen ‘ heute: Grad der Institutionalisierung

und Forschungsdesiderate

9. Kulturgeschichte (Ute Daniel)

1. Kulturgeschichte in der Diskussion I:

Alternativen zur Politikgeschichte

2. Kulturgeschichte in der Diskussion II:

Alternativen zur Sozial- und Gesellschaftsgeschichte

3. Kulturgeschichte in der Diskussion III:

Für ein plurales Theorie- und Methodenverständnis

10. Kultursoziologie (Rainer Winter)

1. Die Aktualität der Kultursoziologie

2. Historische Ursprünge der Kultursoziologie

3. Die Renaissance der Kultursoziologie 209

4. Perspektiven der neueren Forschung

4.1 Kultur und Macht

4.2 Kultur und Konsum

4.3 Kultur und Erlebnis 216

4.4 Kultur und Kommunikation

4.5 Kultur und Globalisierung

5. Schluß

11. Kulturpsychologie und Psychoanalyse als Kulturtheorie (Jürgen Kramer)

1. Kulturvergleichende Psychologie und Kulturpsychologie............... 226

1.1 Vorgeschichte

1.2 Kulturvergleichende Psychologie

1.3 Kulturpsychologie

2. Psychoanalyse als Kulturtheorie

2.1 Die klassische Auffassung.

2.2 Erweiterungen, Differenzierungen, Modifikationen

2.3 Ethnopsychoanalyse und Tiefenhermeneutik 242

3. Perspektiven 
12. Kulturökologie (Peter Finke)

1. Was ist Kulturökologie?

1.1 Naturökologie und Kulturökologie .................................... 248

1.2 Eine junge Disziplin ........................................................ 250

1.3 Konzeptionen der Kulturökologie ........................................ 252

1.4 Vaterfiguren: Uexküll, Bateson, Naess ................................... 255

1.5 Das zentrale Problem: Was ist Kultur? ..................................... 257

2. Grundlagen der Evolutionären Kulturökologie .............................. 258

2.1 Die Ökosysteme des Menschen .............................................. 258

2.2 Strukturelle Erbschaft ........................................................... 259

2.3 Die Ebenen der Kultur ....................................................... 261

2.4 Kulturelle Evolution ........................................................ 262

2.5 Kulturelle Grenzen .......................................................... 263

2.6 Kulturelle Energie ............................................................... 264

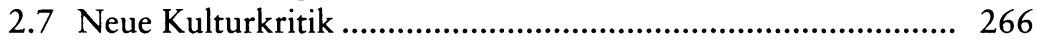

3. Exemplarische Anwendungsfelder ............................................. 267

4. Eine Metapher für Kultur ............................................................. 277

13. Kulturwissenschaftliche Xenologie (Alois Wierlacher und Corinna Albrecht) ................................................................ 280

1. Von der Hermeneutik zur interkulturellen Fremdheitsforschung $\quad 280$

1.1 Die neue Herausforderung: Fremdheitswissen ........................ 280

1.2 Kontur und Gegenstand der Fremdheitsforschung (Xenologie) .......................................................................... 281

1.3 Die Basisdistinktion: Das Andere und das Fremde .................. 284

1.4 Xenologie als Weiterentwicklung der Hermeneutik ................ 285

2. Zur Forschungslage .................................................................... 288

3. Rahmenbegriffe der kulturwissenschaftlichen Xenologie ................ 292

4. Desiderate und Problemfelder der Xenologie ................................ 294

4.1 Problematische Konstitutionsmodi des Fremden ...................... 294

4.2 Fremdheitsforschung als Bedingungsforschung ....................... 295

4.3 Xenologie als Theorie und Geschichte der Gastlichkeit .......... 297

4.4 Zur Xenologie als Wissenschaft vom epistemisch Fremden und vom Neuen .................................................................. 298

4.5 Zur Xenologie der Literatur ............................................... 299

\section{Kulturraumstudien und Interkulturelle Kommunikation}

(Hans-Jürgen Lüsebrink) ................................................................... 307

1. Abgrenzungen und Konvergenzen ................................................ 307

2. Kulturraumstudien ................................................................ 308

2.1 Konzeption und Geschichte .................................................. 308

2.2 Area Studies und Landeskunde ......................................... 310

2.3 Problemfelder ................................................................. 311 
$X \quad$ Inhalt

3. Interkulturelle Kommunikation .................................................... 312

3.1 Interkulturelle Interaktionsprozesse ..................................... 312

3.2 Kulturtransfer .................................................................... 318

3.3 Fremdwahrnehmung .............................................................. 320

3.4 Formen der Interkulturalität: Hybridität, Kreolisierung, Métissage, Néo-Baroque ...................................................... 322

15. Kulturwissenschaften und Geschlechterforschung (Renate Hof) ........ 329

1. Die Ordnung der Geschlechter .................................................... 330

2. Zur Konstruktion des Natürlichen als Text der Kultur ................... 333

3. Das Ende der Geschlechterdifferenz?........................................... 337

4. Die Intelligenz der Kultur ............................................................ 339

5. >Modernisierung der Geisteswissenschaften`? ................................ 341

6. Selbstautorisierung und Performanz ............................................ 344

16. Medienkulturwissenschaft (Siegfried J. Schmidt) ................................ 351

1. Vorbemerkung ……................................................................ 351

2. Ausweichbewegungen .......................................................... 351

3. Medienkonzept ……............................................................... 354

4. Kultur als Programm................................................................ 355

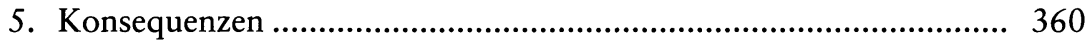

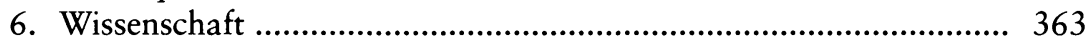

7. Medienkulturwissenschaft ........................................................ 365

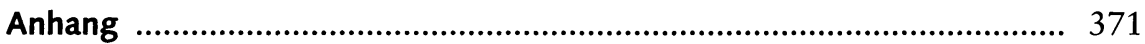

1. Auswahlbibliographie kulturwissenschaftlicher

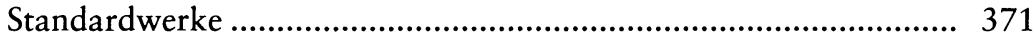

2. Die Autorinnen und Autoren ..................................................... 379

3. Personenregister ...................................................................... 383 